J. Clin. Chem. Clin. Biochem.

Vol. 24, 1986, pp. $651-658$

(C) 1986 by Walter de Gruyter \& Co. Berlin - New York

\title{
Einfache, schnelle, selektive und quantitative Bestimmung von Adrenalin und Noradrenalin im Plasma durch Kombination von Flüssigextraktion, HPLC-Trennung und elektrochemischer Detektion
}

\author{
Von H.-J. Bauch \\ Institut für Arterioskleroseforschung an der Universität Münster \\ U. Kelsch \\ Laboratorium Dr. med. B. Eveld, Essen und \\ W. H. Hauss \\ Institut für Arterioskleroseforschung an der Universität Münster
}

(Eingegangen am 30. August 1985//4. März/26. Mai 1986)

Herrn Professor Dr. U. Gerlach in Dankbarkeit zum 60. Geburtstag gewidmet

Zusammenfassung: Wir beschreiben eine einfache und schnelle Flüssigextraktion zur selektiven und quantitativen Isolierung von Adrenalin und Noradrenalin aus Plasma. Bei der Extraktion wird die Komplexbildung im alkalischen Milieu zwischen Diphenylborsäure und der Diol-Gruppe der Katecholamine sowie IonenpaarBildung ausgenutzt. Die Extraktion gestattet in Verbindung mit HPLC-Trennung und elektrochemischer Detektion die quantitative Bestimmung von Adrenalin und Noradrenalin im Plasma. Diese Methode besitzt die gleiche Spezifität wie die üblicherweise verwendete Adsorption an Aluminiumoxid, zeigt jedoch größere Wiederfindungsraten und Präzision, besonders bei der Bestimmung von Adrenalin.

$A$ rapid, selective änd quantitative determination of epinephrine and norepinephrine from plasma using solventextraction combined with HPLC-separation and electrochemical detection

Summary: A very simple and rapid solvent extraction system for the selective and quantitative isolation of epinephrine and norepinephrine from plasma is described. The extraction system makes use of the complex formation in alkaline medium between diphenylborate and the diol group of the catecholamines in combination with ion-pair formation. The extraction procedure, in conjunction with HPLC-separation and electrochemical detection, allows the quantitative determination of epinephrine and norepinephrine from plasma. This method is as specific as the commonly used adsorption on alumina, but has a much better recovery and even greater precision, especially for the quantitative determination of epinephrine.

\section{Einfiihrung}

Katecholamine sind eine Gruppe von Substanzen, die biogenetisch über die aromatischen Aminosäuren $L$ Phenylalanin bzw. L-3.4-Dihydroxyphenylalanin gebildet werden und die im menschlichen Körper als
Hormone und Neurotransmitter Bedeutung haben. Hierzu zählen Adrenalin, Noradrenalin und Dopamin. Die Konzentration der Katecholamine Adrenalin und Noradrenalin ist im Plasma eine wichtige Kenngröße bei der Klärung pathophysiologi- 
scher, klinischer und pharmakologischer Fragestellungen. Die Konzentration des Noradrenalins im Plasma kann sowohl als Indikator für die Aktivität des sympathischen Nervensystems $(1,2)$ als auch als prognostische Kenngröße bei Patienten mit chronischer kongestiver Herzinsuffizienz gewertet werden (3). Eine wichtige Bedeutung muß den Katecholaminen im Plasma bei der Diagnose und Lokalisation des Phäochromocytoms zuerkannt werden. Bravo et al. haben beispielsweise gezeigt, daß die Bestimmung der Katecholamine im Plasma neben der Bestimmung der Vanillinmandelsäure und der Metanephrine im 24-Stunden-Urin verläßliche Kenngrößen darstellen (4). Ferner lassen Befunde von Dimsdale et al. zum Verlauf der Katecholaminkonzentration im Plasma bei Belastung vermuten, daß der starke Anstieg der Noradrenalin- und Adrenalinkonzentration im Plasma unter Belastung weitreichende Konsequenzen besonders für Patienten mit koronarer Herzkrankheit haben könnte (5).

Zur quantitativen Bestimmung von Katecholaminen im Plasma werden gegenwärtig sowohl radioenzymatische Methoden als auch elektrochemische Verfahren in Verbindung mit Hochleistungsflüssigkeits-Chromatographie (HPLC) angewendet $(6,7)$. Goldstein et al. fanden, daß die Bestimmung der Katecholamine mit Hochleistungsflüssigkeits-Chromatographie und elektrochemischer Detektion genauso verläßlich ist wie deren Bestimmung mittels eines radioenzymatischen Assays, jedoch wegen ihrer einfacheren experimentellen Handhabung und ihrer Schnelligkeit Vorteile bietet (8). Die Anwendung der Hochleistungsflüssigkeits-Chromatographie erfordert eine selektive und nach Möglichkeit quantitative Isolierung der $\mathrm{Ka}$ techolamine aus einer komplexen biologischen Matrix. Vor der chromatographischen Trennung werden die Katecholamine daher oftmals durch selektive Adsorption an Aluminiumoxid aus dem Plasma isoliert und anschließend mit 0,1 mol/1 Perchlorsäure eluiert. Die Auftrennung der einzelnen Katecholamine erfolgt dann mittels HPLC entweder an einer C18-ReversedPhase-Säule oder an einer Ionenaustauscher-Säule bei quantitativer Bestimmung durch Verwendung eines elektrochemischen Detektors (7, 8). Die Adsorption der Katecholamine an Aluminiumoxid ist die am häufigsten angewandte Methode zur Isolierung dieser Substanzen aus der biologischen Matrix. Leider ist dieses Verfahren, abhängig von der Art und Aufbereitung des Aluminiumoxids, starken Schwankungen in Bezug auf die Wiederfindungsrate und die Reproduzierbarkeit unterworfen (9). In der Literatur sind bei Anwendung dieser Methode Wiederfindungsraten für Adrenalin und Noradrenalin zwischen $37 \%$ und $84 \%$ beschrieben worden (10). Smedes et al. entwickelten daher 1982 eine einfache und schnelle Flüssigextraktionsmethode zur selektiven und quantitativen Isolierung von Adrenalin, Noradrenalin und Dopamin aus Plasma und Urin (9). Dieses Verfahren basiert auf einer Komplexbildung zwischen Diphenylborsäure und Katecholgruppen sowie Ionenpaar-Bildung im alkalischen Milieu. Die o. g. Autoren führten unter Anwendung dieser Methode Katecholaminbestimmungen im Plasma lediglich bei fünf gesunden Probanden durch, wobei die gemessenen Adrênalin- und Noradrenalinkonzentrationen im Vergleich zu anderen Literaturangaben im Median erheblich höher waren $(7,9)$.

In der vorliegenden Arbeit sollte zunächst ein Methodenvergleich zur quantitativen Bestimmung der Katecholamine Adrenalin und Noradrenalin unter Anwendung der Aluminiumoxid-Adsorption und der Diphenylborsäure-Extraktion durchgeführt werden. Während die Aluminiumoxid-Adsorptionsmethode häufig zur quantitativen Bestimmung von Adrenalin und Noradrenalin in Plasmaproben bei klinischen Fragestellungen angewendet wurde $(5,8,11)$, sind entsprechende Untersuchungen unter Verwendung der Diphenylborsäure-Extraktionsmethode bislang nicht durchgeführt worden. Um die klinische Anwendbarkeit der Diphenylborsäure-Extraktion zur quantitativen Bestimmung von Adrenalin und Noradrenalin im Plasma auszuloten, wurde daher die Konzentration dieser Substanzen im Plasma bei 19 gesunden Probanden bestimmt.

\section{Material und Methoden}

Blutentnahme zur Bestimmung von Katecholaminkonzentrationen in Ruhe (Basalwerte)

Eine Abbocath-T $18 \mathrm{G}$ Braunüle mit passendem Obturator wurde in eine periphere Vene gelegt und der Proband $30 \mathrm{~min}$ ruhig und flach gelagert. Sodann wurden $10 \mathrm{ml}$ Blut entnommen, mit $1000 \mathrm{i}$. E. Heparin-Natrium und $47 \mu \mathrm{mol}$ reduziertem Glutathion als Antioxidans versetzt und das Plasma durch 15 Minuten langes Zentrifugieren bei $3000 \mathrm{~g}$ gewonnen. AnschlieBend wurde das Plasma bei $-20^{\circ} \mathrm{C}$ gelagert.

Nullplasma

Als Nullplasma wurde entweder eine Lösung von $70 \mathrm{~g} / \mathrm{l} \mathrm{Hu}$ manserumalbumin (Fa. Behring) in $0,15 \mathrm{~mol} / 1$ Natriumchloridlösung oder Poolplasma nach Oxidation verwendet.

Dieses Plasma wurde folgendermaßen hergestellt: 1 Liter Poolplasma wurde mit $9,35 \mathrm{mmol} / 1$ Natrium-meta-perjodat versetzt und $10 \mathrm{~min}$ bei $37^{\circ} \mathrm{C}$ inkubiert. Anschließend wurde das Plasma $15 \mathrm{~min}$ bei $3000 \mathrm{~g}$ zentrifugiert und der Überstand gegen eine $0,15 \mathrm{~mol} / 1$ Natriumchloridlösung dialysiert. Das so behandelte Plasma wurde aliquotiert und bei $-20^{\circ} \mathrm{C}$ gelagert.

Hochleistungsflüssigkeits-Chromatographie (HPLC) und amperometrische Detektion

Die HPLC-Anlage bestand aus einer'M 114 Pumpe (Fa. Beckman), einem M 340 Injektor mit einer 50 pl Probenschleife (Fa. 
Beckman) und einem 641 VA - Elektrochemischen Detektor (Fa. Metrohm). Zur Analytik der Katecholamine wurde eine Nucleosil 7-C18-Säule $(250 \times 8 \times 4 \mathrm{~mm}$; Fa. Macherey \& Nagel) verwendet.

Das Elutionsmittel bestand aus: $62,43 \mathrm{mmol} / \mathrm{l}$ Methansulfonsäure (Fa. Merck); 29,59 mmol/1 Phosphorsäure (Fa. Merck); $0,093 \mathrm{mmol} / 1$ Octansulfonsäure (Kodak); 0,097 mmol/1 EDTA (Titriplex III, Fa. Merck) gelöst in $1000 \mathrm{ml}$ Wasser (Ampuwa ${ }^{\circledR}$ ). Der pH-Wert wurde durch Zugabe von $\mathrm{NaOH}$-Plätzchen auf 2,1 eingestellt. Die Durchflußrate betrug $1,3 \mathrm{ml} / \mathrm{min}$ bei 130 bar Rückdruck und Raumtemperatur.

Die Oxidationsspannung betrug $800 \mathrm{mV}$. Die Messungen wurden bei einer Empfindlichkeit von $1 \mathrm{nA} / \mathrm{V}$ durchgeführt.

Isolierung und Anreicherung der Katecholamine aus Plasmaproben

Zur Isolierung der Katecholamine aus Plasmaproben durch - Adsorption an Aluminiumoxid wurde die von Bauersfeld et al. beschriebene Methode angewendet (11). Zur Elution der Katecholamine wurde jedoch eine 0,1 mol/1 Perchlorsäure-Lösung verwendet. Das verwendete Aluminiumoxid (Fa. Waters) war ein speziell für die Katecholaminanalytik gereinigtes, käuflich erwerbliches Präparat, das laut Angabe der Herstellerfirma nicht weiter nachgereinigt zu werden braucht.

Zur Isolierung der Katecholamine aus Plasmaproben durch Flüssigextraktion wurde die von Smedes et al. (9) beschriebene Methode in modifizierter Form angewendet, die daher im Folgenden kurz beschrieben wird. Zunächst wurden folgende Lösungen hergestellt:

Lösung A: $2 \mathrm{~mol} / 1$ Ammoniumchlorid-/AmmoniumhydroxidLösung (Fa. Merck);

$8 \mathrm{mmol} / \mathrm{l}$ Diphenylborsäure-Ethanolaminkomplex (ICN Pharmaceuticals);

13,4 mmol/1 EDTA (Titriplex III, Fa. Merck); pH 8,5 mit konz. Salzsäure einstellen.

Lösung B: 4,57 mmol/1 Tetra-octyl-ammoniumbromid (Fluka) werden in $250 \mathrm{ml}$ Chloroform p. A. gelöst und mit $750 \mathrm{ml} n$-Heptan p. A. gemischt.

Lösung C: $n$-Octanol (Fa. Merck)

Lösung D: 0,2 mol/1 Essigsäure (Fạ. Merck)

Je nach Versuch wurden mindestens $2,0 \mathrm{ml}$, jedoch maximal 4,0 ml Plasma mit 1,0 ml Lösung $A$ und 5,0 ml Lösung $B$ versetzt und 3-5 min auf einem Vortex-Mixer gut durchgemischt. Von der oberen (organischen) Phase wurden 4,0 ml abgenommen, in ein spitż zulaufendes Glaszentrifugenröhrchen gegeben und mit $2 \mathrm{ml}$ Lösung $C$ und $100 \mu$ Lösung D versetzt. Das Gemisch wurde wiederum 3-5 min kräftig geschüttelt. Anschließend wurde von der unteren essigsauren Phase ein Aliquot mit einer Hamiltonspritze entnommen und in das HPLC-System zur weiteren Analyse injiziert.

Das obën beschriebene Extraktionsverfahren unterscheidet sich im Wesentlichen von der von Smedes et al. (9) beschriebenen Methode dadurch, daß zur Komplexbildung die doppelte Menge an Diphenylborsäure-Ethanolaminkomplex verwendet wurde. Außerdem wählten Smedes et al. zur Extraktion der Katecholamine eine Lösung bestehend aus $n$-Hexan mit $1 \%$ Octanolzusatz und $4,57 \mathrm{mmol} / 1$ Tetra-octyl-ammoniumbromid (vgl. Lösung B).

\section{Stammlösungen}

Von den einzelnen Katechol-Derivaten wurden Stammlösungen mit einer Konzentration von $5,5 \mathrm{mmol} / 1$ in $0,2 \mathrm{~mol} / 1$ Essigsäure hergestellt. Diese Lösungen wurden bei $-20^{\circ} \mathrm{C}$ tiefgefroren gelagert. Sie waren über ein Jahr lang stabil.

\section{Quantitative Bestimmungen}

Die Konzentration der Katecholamine wurde durch Messung der Peakhöhe und Vergleich mit der Peakhöhe einer entsprechenden. Standardlösung ermittelt.

\section{Ergebnisse}

HPLC von Katechol-Derivaten auf einer Reversed-Phase C18-Säule

Voraussetzung für eine exakte quantitative Bestimmung von Katecholaminen sind gute chromatographische Bedingungen, die eine saubere Trennung verschiedener Katechol-Derivate gewährleisten. Denn neben Adrenalin und Noradrenalin befinden sich im Plasma weitere Katechol-Derivate, die entweder biogenetische Vorstufen oder aber Abbauprodukte der o. g. Katecholamine darstellen. Wie Abbildung 1 zeigt, gewährleisten die gewählten Bedingungen eine gute HPLC-Trennung von Adrenalin, Noradrenalin und anderen Katechol-Derivaten, die im Plasma vorhanden sein können (Peak No. 1-3; 5-9). Das 3.4Dihydroxybenzylamin (Peak No. 4) ist eine Substanz, die bei der quantitativen Bestimmung von Katecholaminen gern als interner Standard verwendet wird.

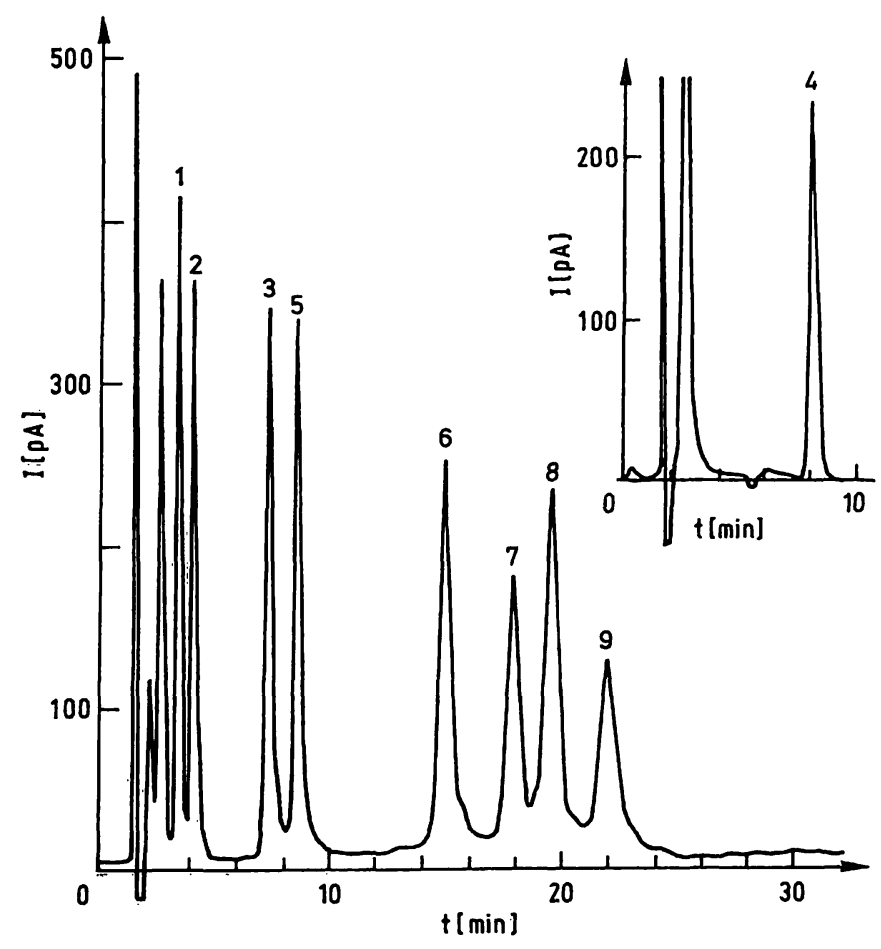

Abb. 1. HPLC-Trennung verschiedener Katechol-Derivate an einer Nucleosil 7-C18-Säule und elektrochemischer Detektion

1: 3.4-Dihydroxyphenylserin,

2: Noradrenalin,

3: Adrenalin,

4: 3.4-Dihydroxybenzylamin,

5: 5-Hydroxydopamin,

6: Dopamin,

7: 3.4-Dihydroxyphenylalanin,

8: 3.4-Dihydroxyphenylessigsäure,

9: Epinin 
Die lineare Beziehung zwischen Peakhöhe und der Konzentration der eingesetzten Substanz wurde für die Katechol-Derivate 3.4-Dihydroxyphenylserin, Noradrenalin, Adrenalin, 3.4-Dihydroxybenzylamin, 5-Hydroxydopamin, Dopamin, 3.4-Dihydroxyphenylalanin, 3.4-Dihydroxyphenylessigsäure und Epinin für den Konzentrationsbereich von 2,5 nmol/1-500 nmol/1 überprüft. Dazu wurden 4-fach Messungen mit 11 verschiedenen Konzentrationen im o. g. Bereich durchgeführt. Die zweidimensionale Verteilung der Peakhöhen und der jeweils vorgegebenen Konzentrationen zeigte eine lineare Abhängigkeit im gesamten Meßbereich. Die Daten der linearen Regressionsanalyse sind in Tabelle 1 wiedergegeben, wobei die vorgegebenen Konzentrationen als unabhängige und die Peakhöhen als abhängige Variable gewählt wurden. Die Standardabweichung der Residuen ist bei allen Katechol-Derivaten klein. Daraus ist ersichtlich, daß die einzelnen Meßpunkte nur geringfüging um die Regressionsgerade streuen.

Vergleich zweier Aufarbeitungsmethoden zur Isolierung und Quantifizierung von Katecholaminen aus Plasmaproben

Eine Humanserumalbumin-Lösung (Nullplasma), die jeweils 0,137 nmol/1 Adrenalin, 0,117 nmol/1 3.4-Dihydroxyphenylserin, $1,478 \mathrm{nmol} / 1$ Noradrenalin und 1,137 nmol/1 3.4-Dihydroxybenzylamin enthielt, wurde mittels Aluminiumoxid-Adsorption und Diphenylborsäure-Extraktion aufgearbeitet und mittels HPLC mit elektrochemischer Detektion analysiert. Das Ergebnis ist in Abbildung 2 dargestellt.

Die Probe, die mittels Aluminiumoxid-Adsorption aufgearbeitet wurde, zeigt im Chromatogramm neben den eingesetzten Kacholaminen Adrenalin, Noradrenalin, Dihydroxyphenylserin und Dihydroxybenzylamin einige Störsubstanzen, die besonders die Bestimmung von Adrenalin und Dihydroxybenzylamin beeinträchtigen (s. Abb. 2a). Außerdem bedingt das

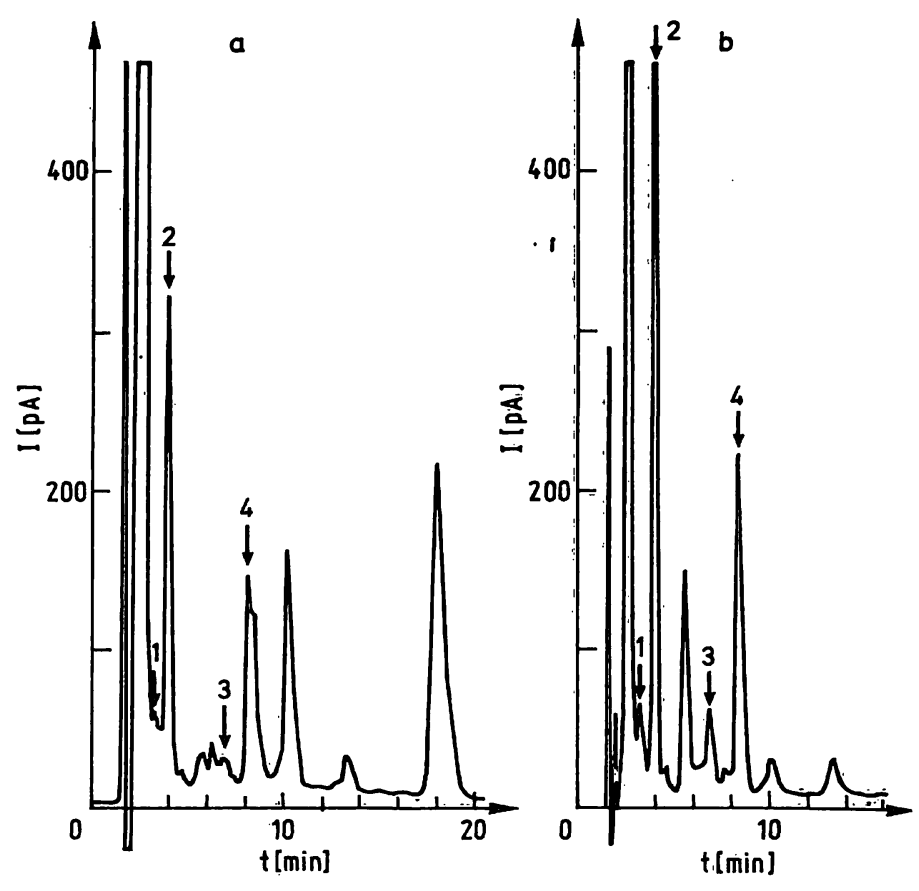

Abb. 2. Chromatogramme einer aufgestockten Probe „Nullplasma“, die mittels Aluminiumoxid-Ädsorption (a) und Diphenylborsäure-Extraktion (b) aufgearbeitét wurde;

$1=3.4$-Dihydroxyphenylserin,

$2=$ Noradrenalin,

$3=$ Adrenalin,

4 = 3.4-Dihydroxybenzylamin

Auftreten einer Störsubstanz im Chromatogramm nach 19 min verhältnismäßig lange Analysenzeiten. Die Probe dagegen, die mittels DiphenylborsäureExtraktion aufgearbeitet wurde, zeigt ein sebr sauberes, verhältnismäßig peakarmes Chromatogramm, das eine gute quantitative Bestimmung der eingesetzten Katecholamine gestattet (s. Abb. 2 b). Die Analysenzeit ist wesentlich kürzer, da nach einer Laufzeit von $14 \mathrm{~min}$ keine weiteren Substanżen beôbachtet wurden. Außerdem ist die Wiederfindungsrate augenscheinlich erheblich größer bei Extraktion der Katecholamine mit Diphenylborsäure als bei der Isolierung durch Adsorption an Aluminiumoxid.

Tab. 1. Lineare Regressionsanalyse zur Beziehung zwischen Konzentration und Peakhöhe bei neun Katechol-Derivaten für den Konzentrationsbereich von $2,5-500 \mathrm{nmol} / 1$.

\begin{tabular}{|c|c|c|c|c|}
\hline Substanz & $\begin{array}{l}\text { Korrelations- } \\
\text { koeffizient } \\
\text { r }\end{array}$ & $\begin{array}{l}\text { Anstieg } \\
\text { (b) } \\
\left(\mathrm{cm} / \mathrm{nmol} \cdot 1^{-1}\right)\end{array}$ & $\begin{array}{l}\text { Achsen- } \\
\text { abschnitt (a) } \\
\text { (cm) }\end{array}$ & $\begin{array}{l}\text { Standard- } \\
\text { abweichung } \\
\text { der Residuen }\end{array}$ \\
\hline $\begin{array}{l}\text { 3.4-Dihydroxyphenylserin } \\
\text { Noradrenalin } \\
\text { Adrenalin } \\
\text { 3.4-Dihydroxybenzylamin } \\
\text { 5-Hydroxydopamin } \\
\text { Dopamin } \\
\text { 3.4-Dihydroxyphenylalanin } \\
\text { 3.4-Dihydroxyphenylessigsäure } \\
\text { Epinin }\end{array}$ & $\begin{array}{l}1,000 \\
1,000 \\
1,000 \\
1,000 \\
1,000 \\
1,000 \\
1,000 \\
1,000 \\
0,999\end{array}$ & $\begin{array}{l}0,464 \\
0,408 \\
0,257 \\
0,268 \\
0,268 \\
0,199 \\
0,128 \\
0,152 \\
0,102\end{array}$ & $\begin{array}{r}0,067 \\
-0,057 \\
0,014 \\
0,022 \\
0,029 \\
0,004 \\
-0,044 \\
-0,073 \\
-0,025\end{array}$ & $\begin{array}{l}1,069 \\
0,889 \\
0,792 \\
1,121 \\
1,043 \\
0,843 \\
0,493 \\
0,807 \\
0,677\end{array}$ \\
\hline
\end{tabular}




\section{Vergleich der Wiederfindungsraten}

Das o. g. „Nullplasma“ wurde mit Dihydroxyphenylserin, Noradrenalin, Adrenalin und Dihydroxybenzylamin versetzt und nach beiden Methoden entsprechend aufgearbeitet. Die eingesetzten Substanzmengen variierten beim 3.4-Dihydroxyphenylserin im Bereich von $0,117-0,702 \mathrm{nmol} / \mathrm{l}$, beim Noradrenalin im Bereich von 1,478-8,868 $\mathrm{nmol} / \mathrm{l}$, beim Adrenalin im Bereich von $0,137-0,822 \mathrm{nmol} / \mathrm{l}$ und beim Dihydroxybenzylamin betrug die Konzentration stets 1,137 $\mathrm{nmol} / \mathrm{l}$ in jeder Probe. Jede Probe mit gleicher Zusammensetzung wurde viermal analysiert. Tabelle 2 zeigt einige statistische Berechnungen zur Wiederfindungsrate bei der quantitativen Bestimmung verschiedener Katecholamine bei Aufarbeitung der Plasmaproben mittels Aluminiumoxid-Adsorption bzw. Diphenylborsäure-Extraktion. Die Wiederfindungsraten sind für die einzelnen Katecholamine bei beiden Methoden individuell verschieden. Sie sind bei der Aufarbeitung mit Aluminiumoxid durchweg erheblich kleiner im Vergleich zur Extraktion mit Diphenylborsäure. Beim Arbeiten mit Aluminiumoxid beträgt die durchschnittliche Wiederfindungsrate für 3.4-Dihydroxyphenylserin $37 \%$, für Noradrenalin $49,2 \%$, für Adrenalin 44,0\% und für 3.4-Dihydroxybenzylamin $56,3 \%$. Bei Anwendung der Diphenylborsäure-Extraktion wurde für die Katecholamine Adrenalin und Noradrenalin praktisch kein Substanzverlust bei der Aufarbeitung beobachtet, während die durchschnittlichen Wiederfindungsraten beim 3.4-Dihydroxyphenylserin mit $96,5 \%$ und $93,0 \%$ beim Dihydroxybenzylamin etwạs geringer waren. Die Variationskoeffi- zienten (VK) sind bei beiden Aufarbeitungsmethoden für alle getesteten Noradrenalinkonzentrationen durchweg kleiner als $3 \%$. Bei der Aufarbeitung von Plasmaproben mit geringer Adrenalinkonzentration, $0.137 \mathrm{nmol} / \mathrm{l}$, beträgt der Variationskoeffizient für die Wiederfindungsrate bei Anwendung der Aluminiumoxid-Adsorption $12,0 \%$, bei Extraktion mit Diphenylborsäure dagegen nur $6,58 \%$. Bei größeren Adrenalinkonzentrationen sind die einzelnen Variationskoeffizienten bei beiden Methoden $\leqslant 5,02 \%$ (s. Tab. 2).

\section{Vergleich beider Bestimmungsmethoden}

Poolplasma wurde nach Oxidation mit verschiedenen Adrenalin- und Noradrenalinmengen versetzt. Die vorgegebenen Adrenalinkonzentrationen lagen zwischen $0,137-0,822 \mathrm{nmol} / \mathrm{l}$, während die Noradrenalinkonzentrationen $1,478-8,868 \mathrm{nmol} / \mathrm{l}$ betrugen. Außerdem enthielt jede Plasmaprobe $1,137 \mathrm{nmol} / 1$ Dihydroxybenzylamin als internen Standard. Die Plasmaproben wurden mittels Aluminiumoxid-Adsorption und Diphenylborsäure-Extraktion aufgearbeitet. Die nach beiden Methoden ermittelten Konzentrationen wurden mit den eingewogenen Mengen durch lineare Regression verglichen. Die Ergebnisse sind in Tabelle 3 dargestellt.

Wie aus Tabelle 3 hervorgeht, konnte auf dem 5\%Niveau kein signifikanter Unterschied zwischen den vorgegebenen Mengen an Adrenalin und Noradrenalin und den nach Diphenylborsäure-Extraktion bestimmten Konzentrationen festgestellt werden. Der

Tab. 2. Statistische Berechnungen zur Ẅiederfindungsrate bei der quantitativen Bestimmung verschiedener Katecholamine unter Verwendung von zwei verschiedenen Aufarbeitungsmethoden.

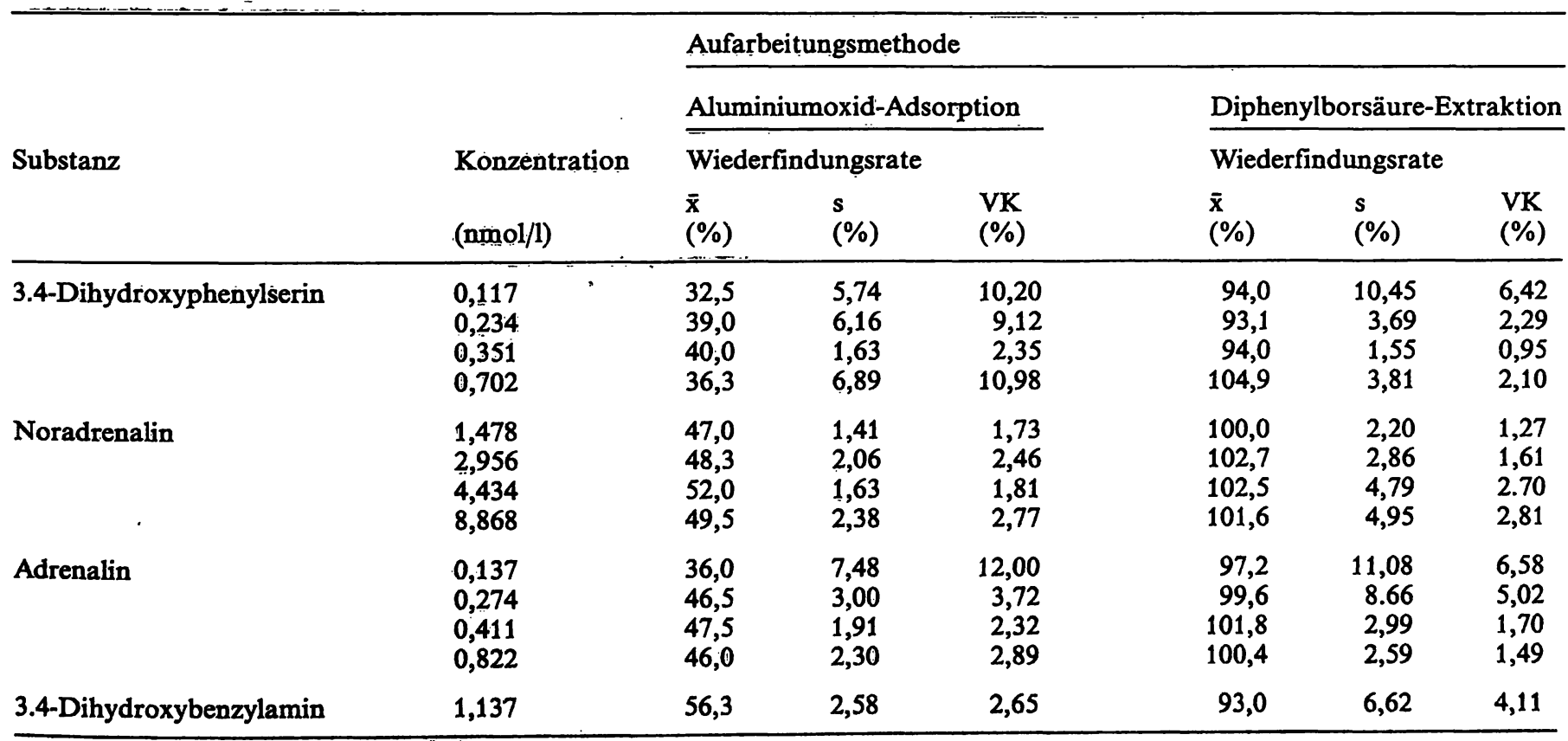


Tab. 3. Methodenvergleich durch lineare Regressionsanalyse zur quantitativen Bestimmung von Adrenalin und Noradrenalin in Plasmaproben mittels Aluminiumoxid-Adsorption und Diphenylborsäure-Extraktion.

\begin{tabular}{|c|c|c|c|c|c|c|}
\hline \multirow{2}{*}{$\begin{array}{l}\text { Substanz } \\
\text { Methode }\end{array}$} & \multicolumn{3}{|l|}{ Adrenalin } & \multicolumn{3}{|c|}{ Noradrenalin } \\
\hline & Vorgegeben & $\begin{array}{l}\text { Aluminiumoxid- } \\
\text { Adsorption }\end{array}$ & $\begin{array}{l}\text { Diphenyl- } \\
\text { borsäure- } \\
\text { Extraktion }\end{array}$ & Vorgegeben & $\begin{array}{l}\text { Aluminiumoxid- } \\
\text { Adsprption }\end{array}$ & $\begin{array}{l}\text { Diphenyl- } \\
\text { borsäure- } \\
\text { Extraktion }\end{array}$ \\
\hline Anzahl der Meßwerte & 16 & 16 & 16 & 16 & 16 & 16 \\
\hline$c_{\min }(\mathrm{nmol} / \mathrm{l})$ & 0,137 & 0,080 & 0,131 & 1,478 & 1,240 & 1,405 \\
\hline$c_{\max }(\mathrm{nmol} / \mathrm{l})$ & 0,822 & 0.680 & 0,827 & 8,868 & 7,861 & 9,086 \\
\hline & 0,411 & 0,326 & 0,411 & 4,434 & 3,851 & 4,500 \\
\hline t-Test $(\bar{x}-\bar{x}$ vorgegeben $)$ & - & $\mathrm{p}<0,01$ & n. s. & - & $p<0,001$ & n. s. \\
\hline Anstieg b & - & 0,325 & 1,009 & - & 0,870 & 1,007 \\
\hline$t$-Test $(b=1)$ & - & $\mathrm{p}<0,001$ & n. s. & - & $p<0,001$ & n. $\mathbf{s}$. \\
\hline Achsenabschnitt a & - & $-0,011$ & $-0,002$ & - & $-0,006$ & 0,035 \\
\hline $\begin{array}{l}\text { Standardabweichung } \\
\text { der Residuen }\end{array}$ & - & 0,015 & 0,005 & - & 0,072 & 0,130 \\
\hline
\end{tabular}

Anstieg der Regressionsgeraden unterschied sich nicht signifikant von 1. Bei Anwendung der Aluminiumoxid-Adsorption konnte ein signifikanter Unterschied zwischen den vorgegebenen Katecholaminmengen und den ermittelten Konzentrationen an Adrenalin- und Noradrenalin beobachtet werden. Bei Anwendung dieser Methode unterschied sich der Anstieg der Regressionsgeraden signifikant von 1. Die methodisch bestimmten Konzentrationen waren durchweg zu niedrig.

Vergleicht man die quantitativen Bestimmungen von Adrenalin, Noradrenalin und Dihydroxyphenylserin bei beiden Aufarbeitungsmethoden direkt miteinander, so erhält man bei Berechnung der Korrelationskoeffizienten $r$ für Adrenalin $r=0,98$, Noradrenalin $r=0,99$ und Dihydroxyphenylserin $r=0,98$. Dies zeigt, da $\beta$ beide Aufarbeitungsmethoden eine vergleichbare Substanzgruppenspezifität besitzen.

Präzision und Nachweisgrenze der quántitativen Bestimmung von Adrenalin und Noradrenalin in Plasmaproben mittels Diphenylborsäure-Extraktion

Die Reproduzierbarkeit dieser Methode wurde durch wiederholte Bestimmungen (n) unter Verwendung eines Poolplasmas, das $0,137 \mathrm{nmol} / 1$ Adrenalin und $1,478 \mathrm{nmol} / 1$ Noradrenalin enthielt, überprüft. Für die Intra-Assay-Präzision wurden 10 Bestimmungen durchgeführt. Die Variantionskoeffizienten (VK) betrugen für Adrenalin VK $=3,8 \%$ und für Noradrenalin VK $=1,7 \%$. Die Inter-Assay-Präzision, gemessen an 15 Tagen, ergab für Adrenalin VK $=7,2 \%$ und für Noradrenalin VK $=4,7 \%$ (s. Tab. 4).

Bei Aufarbeitung von $2,0 \mathrm{ml}$ Plasma und elektrochemischer Detektion mit einer Empfindlichkeit von 0,5
Tab. 4. Präzision der quantitativen Bestimmung von Adrenalin und Noradrenalin mittels Diphenylborsäure-Extraktion.

\begin{tabular}{llll}
\hline & & Adrenalin & Noradrenalin \\
\hline Intraassay & $\mathrm{N}$ & 10 & 10 \\
& $\overline{\mathrm{x}}(\mathrm{nmol} / \mathrm{l})$ & 0,135 & 1,490 \\
& $\mathrm{~s}(\mathrm{nmol} / \mathrm{l})$ & 0,0051 & 0,025 \\
& $\mathrm{VK}(\%)$ & 3,8 & 1,7 \\
Interassay & $\mathrm{N}$ & 15 & 15 \\
& $\overline{\mathrm{x}}(\mathrm{nmol} / \mathrm{l})$ & 0,134 & 1,506 \\
& $\mathrm{~s}(\mathrm{nmol} / \mathrm{l})$ & 0,0096 & 0,071 \\
& $\mathrm{VK}(\%)$ & 7,2 & 4,7 \\
\hline
\end{tabular}

$\mathrm{nA} / \mathrm{V}$ lag die Nachweisgrenze für Adrenalin bei 0,082 $\mathrm{nmol} / 1$ (气 $15 \mathrm{ng} / \mathrm{l})$ und für Noradrenalin $0,053 \mathrm{nmol} / \mathrm{l}$ ( $\cong 9 \mathrm{ng} / \mathrm{l})$. Unter dièsen Bedingungen warr die Peakhöhe größer als $1 \mathrm{~cm}$ und der Variationskoeffizient war für beide Substanzen kleiner als $10 \%$.

Quantitative Bestimmung von Adrenalin und Noradrenalin im Plasma bei gesunden Probanden

Unter Anwendung der Diphenylborsäure-Extraktion wurde im Folgenden geprüft, ob diese Methode für eine routinemäßige Bestimmung von Adrenalin und Noradrenalin im Plasma bei klinischen Fragestellungen geeignet ist.

Abbildung 3 zeigt das Chromatogramm einer Plasmaprobe von einem gesunden Probanden. Die Katecholamine Adrenalin und Noradrenalin sind gut quantifizierbar. Die gefundenen Konzentrationen betrugen für Adrenalin 0,147 nmol/1 und für Noradrenalin $1,495 \mathrm{nmol} / \mathrm{l}$. 


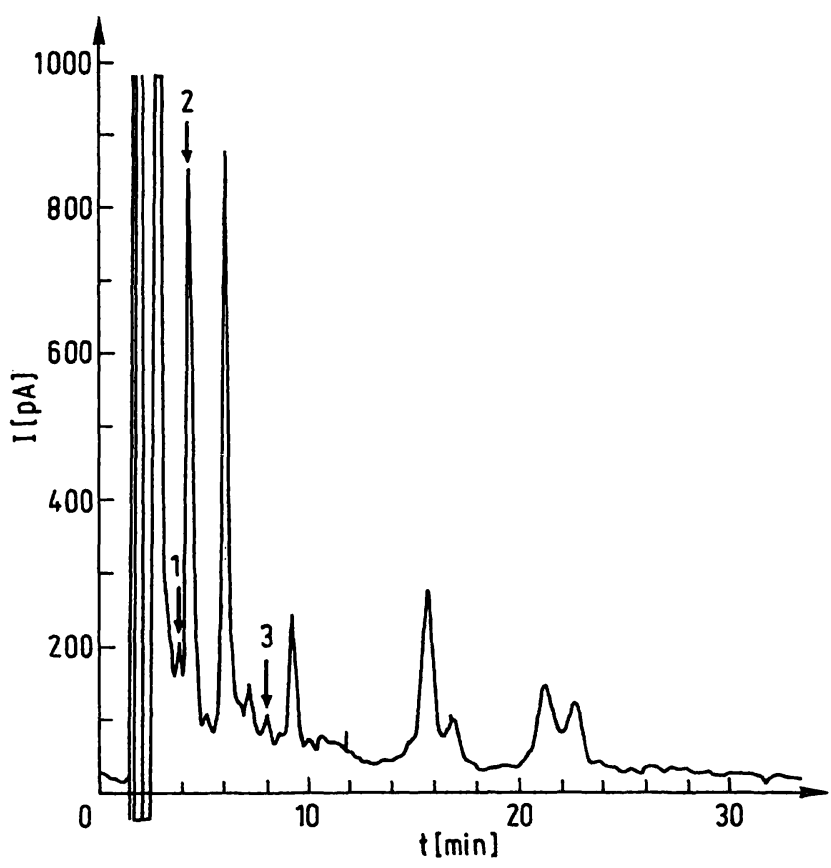

Abb. 3. Chromatogramm einer Plasmaprobe von einem gesunden Probanden;

1 = 3.4-Dihydroxyphenylserin,

2 = Noradrenalin,

3 = Adrenalin

Um festzustellen, welche Konzentrationen von Adrenalin und Noradrenalin im Plasma durchschnittlich gefunden werden, wurden Plasmaproben von 19 gesunden Probanden unter Anwendung der Diphenylborsäure-Extraktion aufgearbeitet und mittels HPLC mit elektrochemischer Detektion analysiert. Die durchschnittlich gefundenen Konzentrationen im Plasma betrugen für Adrenalin 0,164 $\pm 0,071 \mathrm{nmol} / 1$ und für Noradrenalin $2,861 \pm 1,200 \mathrm{nmol} / \mathrm{l}$.

\section{Diskussion}

Die vorliegenden Ergebnisse zeigen, daß die Flüssigextraktion von Katecholaminen aus Plasmaproben mit Diphenylborsäure im Vergleich zur bislang üblicherweise angewendeten Aluminiumoxid-Adsorption Vorteile bietet. Die Probenaufbereitung ist bei Anwendung der Flüssigextraktion hinsichtlich der experimentellen Handhabung wesentlich einfacher, schneller und leichter automatisierbar. Die Berechnung der Korrelationskoeffizienten zeigt, daß das Verfahren für Katecholamine eine vergleichbare Substanzgruppenspezifität wie die Aluminiumoxid-Adsorption bei erheblich besseren Wiederfindungsraten besitzt. Die Wiederfindungsraten sind bei Anwendung der Aluminiumoxid-Adsorption für die einzelnen Katecholamine individuell verschieden. Mit dem verwendeten, speziell für die Katecholaminanalytik gereinigten, käuflichen Aluminiumoxid wurden durchschnittliche Wiederfindungsraten von $37 \%$ für 3.4-Dihydroxyphenylserin, $49,2 \%$ für Noradrenalin, $44,0 \%$ für Adrenalin und 56,3\% für 3.4-Dihydroxybenzylamin erzielt. $\mathrm{Da}$ die Wiederfindungsrate für das 3.4-Dihydroxybenzylamin, das als interner Standard verwendet wurde, deutlich größer war als die Wiederfindungsrate für die zu bestimmenden Katecholamine Adrenalin und Noradrenalin, müssen die bei der quantitativen Bestimmung ermittelten Konzentrationen für beide Verbindungen zu gering sein. Dies wurde beim Methodenvergleich, quantitative Bestimmung von Adrenalin und Noradrenalin mittels Aluminiumoxid-Adsorption und DiphenylborsäureExtraktion, durch die Daten der linearen Regressionsanalyse bestätigt. Die nach der AluminiumoxidAdsorptionsmethode bestimmten Konzentrationen für Adrenalin und Noradrenalin waren durchweg geringer als die im Poolplasma vorgegebenen, während die nach der Diphenylborsäure-Extraktion ermittelten Konzentrationen sich nicht signifikant von den vorgegebenen unterschieden. Diese Ergebnisse zeigen, daß die Verwendung eines internen Standards, der zur Ermittlung der Wiederfindungsrate bei der Aluminiumoxid-Adsorptionsmethode unbedingt notwendig ist, bei der quantitativen Bestimmung von Adrenalin und Noradrenalin zu einem individuell verschiedenen Meßfehler führen kann. Es wäre zu prüfen, ob eine Nachreinigung des verwendeten Aluminiumoxids nach der Vorschrift von Anton \& Sayre (12) zu einheitlichen Wiederfindungsraten bei den $0 . \mathrm{g}$. Katecholaminen führt. Diese Probleme traten bei Anwendung der Flüssigextraktion nicht auf. Die Wiederfindungsraten lagen ziemlich konstant bei $\pm 100 \%$ für Adrenalin und Noradrenalin, so daß bei Anwendung dieser Methode auf eine interne Standardisierung bei der quantitativen Bestimmung verzichtet werden kann. In den Chromatogrammen, die durch Kombination der beschriebenen Extraktionsmethode mit Hochleistungsflüssigkeitschromatographie und elektrochemischer Detektion erstellt wurden, traten weniger Störsubstanzen auf als bei der Aluminiumoxid-Adsorptionsmethode. Daraus ergaben sich kürzere Analysenzeiten.

Die Bestimmung von Adrenalin und Noradrenalin in Plasmaproben zur Beantwortung klinischer Fragestellungen lieferte unter Verwendung der Diphenylborsäure-Extraktionsmethode brauchbare Ergebnisse. Bei 19 gesunden Probanden wurden im Plasma mit Hilfe dieser Methode Konzentrationen von 0,164 $\pm 0,071 \mathrm{nmol} / \mathrm{l}$ für Adrenalin und 2,861 $\pm 1,200$ nmol/1 für Noradrenalin gemessen. Diese Werte sind niedriger als die von Smedes et al. publizierten Konzentrationen für Adrenalin $(0,682 \pm 0,033 \mathrm{nmol} / \mathrm{l})$ 
und Noradrenalin $(3,047 \pm 0,101 \mathrm{nmol} / \mathrm{l})$ im Plasma gesunder Probanden (9). Die von uns gemessenen Adrenalinwerte stimmen mit den Literaturangaben von Cryer \& Hjemdahl überein, während die Noradrenalinwerte durchschnittlich etwas höher liegen (7, 13). Weitere Messungen zùr Katecholaminsyn- these bei der Diagnostik eines Phäochromocytoms und unter ergometrischer Belastung unterstreichen die Anwendbarkeit der Diphenylborsäure-Extraktion zur Bestimmung von Adrenalin und Noradrenalin in Plasmaproben bei der Bearbeịtung klinischer Fragestellungen (14).

\section{Literatur}

1. Goldstein, D. S. (1981) Am. J. Cardiol. 48, 1147-1154.

2. Lake, C. R., Chernow, B., Goldstein, D. S., Glass, D. G., Coleman, M. \& Ziegler, M. G. (1984) Federation Proc. 43, $52-56$.

3. Cohn, J. N., Levine, T. B., Olivary, M. T., Garberg, V., Lura, D. \& Francis, G. S. (1984) N. Engl. J. Med. 311, $819-823$.

4. Bravo, E. L., Tarazi, R. C., Gifford, R. W. \& Stewart, B. H. (1979) N. Engl. J. Med. 301, 682-686.

5. Dimsdale, J. E., Hartley, L. H., Guiney, T., Ruskin, J. N. \& Greenblatt, D. (1984) J. Am. Med. Ass. 251, 630-632.

6. Da Prada, M. \& Zürcher, G. (1976) Life Sci. 19, $1161-1174$.
7. Hjemdahl, P. (1984) Am. J. Physiol. 247. E13-E20.

8. Goldstein, D. S., Feuerstein, G., Izzo, J. L., Kopin, I. J. \& Keiser, H. R. (1981) Life Sci. 28, 467-475.

9. Smedes, F., Kraak, J. C. \& Poppe, H. (1982) J. Chromatog. $231,25-39$.

10. Bauersfeld, W. (1984) Dissertation, Stuttgart.

11. Bauersfeld, W., Ratge, D., Knoll, E. \& Wisser, H. (1984) Fresenius Z. Anal. Chem. 317, 679-680.

12. Anton, A. H. \& Sayre, D. F. (1962) J. Pharmacol. Exp. Therapeut. 138, 360-374.

13. Cryer, P. E. (1980) N. Engl. J. Med. 303, 436-444.

14. Bauch, H.-J., Kelsch, U. \& Hauss, W. H., unveröffentlichte Ergebnisse.
Dr. Hans-Joachim Bauch Institut für Arterioskleroseforschung an der Universität Münster Domagkstr. 3 D-4400 Münster 